Bull.Fac, Agric.,Cairo Univ.,62: 192-203 (2011).

\title{
EFFECT OF MINERAL FERTILIZERS (NITROGEN AND PHOSPHORUS) AND BIOLOGICAL FERTILIZER (NITROBIEN) ON GROWTH AND YIELD OF GARLIC
}

(Received: 6. 11. 2010)

\author{
By \\ Kh. A. Omer and K.B. Esho
}

Horticulture and Landscape Design, College of Agriculture and Forestry, Mosul University, Iraq.

\begin{abstract}
The experiment was carried out in the Vegetable Field, Horticulture Department, College of Agriculture and Forestry, Mosul University, Iraq for two autumn seasons of 2007/2008 and 2008/2009, to study the effect of levels of nitrogen and phosphorus fertilizer $(0,0)(25,50)(50,100)$ and $(75,150)$ $\mathrm{kg}$ /donum with or without the biological fertilizer (Nitrobien) .

The experiment involved eight treatments and the Complete Randomized Block Design (C.R.B.D) was used with three replicates .The data showed that there was significant increase in the vegetative growth characteristics and for the two growing seasons as a result of fertilization with nitrogen and phosphorus and also with nitrobien, and the interaction of them.The treatment showed that there was a significant increase in the characteristics of head of the garlic ( weight, diameter and height), and in the numbers of bulbs/head .The interaction between $(50,100)$ levels of nitrogen and phosphorus with biological fertilizer (Nitrobien) gave the best result in both of the two seasons and also this treatment gave the best significant increase in characteristic quality of bulbs (weight, length, and diameter) and also in T.S.S and the percentage of dry matter in bulbs in both two seasons. The interaction between $(50,100)$ levels of nitrogen and phosphous with Nitrobien gave the highest total yield per unit which was 12.32 and 10.20 ton/hectar in both two seasons, respectively .
\end{abstract}

Key words: biological fertilizer, garlic, mineral fertilizer, nitrobien.

\footnotetext{
تأثير التسميد المعدنى (النتروجينى والفوسفورى) والسماد الحيوى (النتروبين) فى نمو وحاصل الثوم خالدة عبد الله عمر - كمال بنيامين ايثو$$
\text { قسم البستنة و هندسة الحدائق -كلية الزر اعة و الغابات- جامعة الموصل ـ العراق }
$$

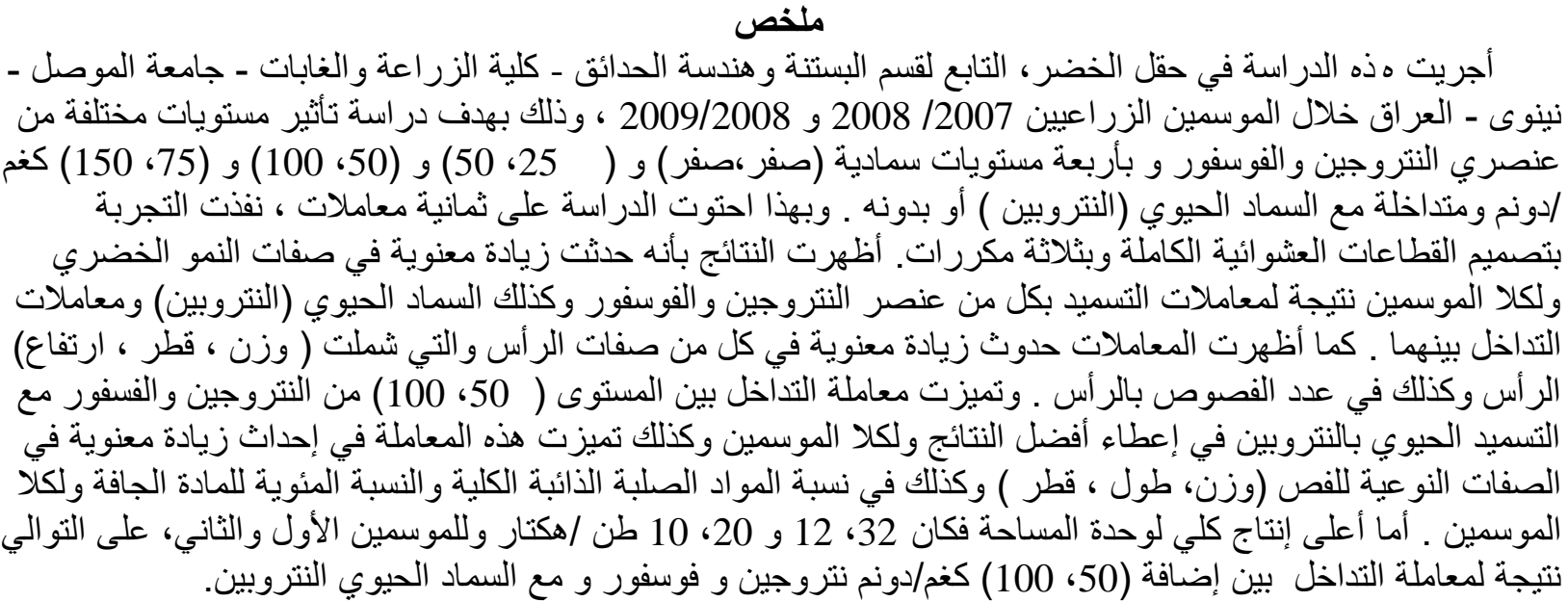


حيوي ومثبت للنتروجين ويحتوي على بكتريا الازوتوباكتر المثبتة للنتروجين و هي من نوع البكتريا اللا تكافلية ـ وقد التد

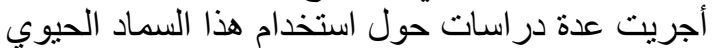
ومتداخلاً مع الأسمدة المعدنية في مجال إنتاج الخضر ومناد النها

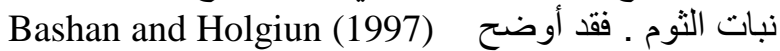

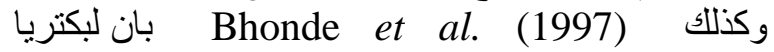
الازوتوباكتر دور مهم في نمو نبات الثوم وذللك من خلال

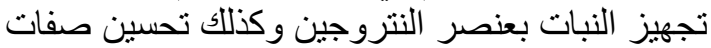

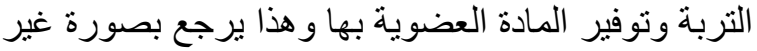

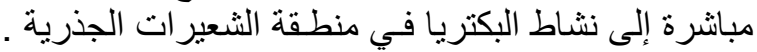

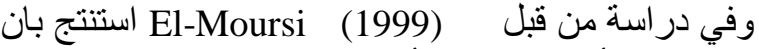
المعاملة بالأسمدة الحيوية أدت إلى زيادة نسبة العناصر

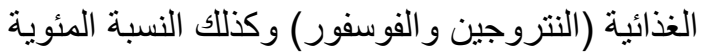

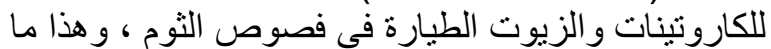
أكده(El-Shabasi et al., 2003)، أما (2001 ) فقد درس تأثير كل من التلقيح بالأسمدة الحيوية مع التسميد

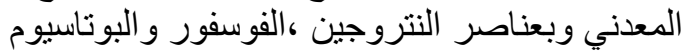

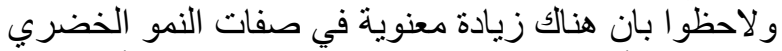

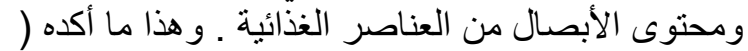

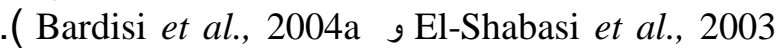
ومن خلال مر اجعتنا للار اسات والبحوث حول إنتاج الثوم

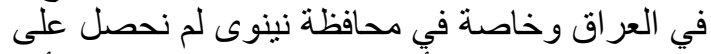

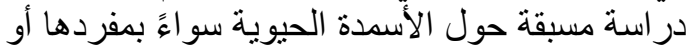

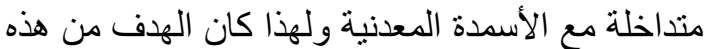
الدراسة هو لمعرفة دور السماد الحئدة الحيوي النتروبين ومتداخلاً

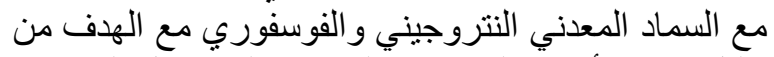

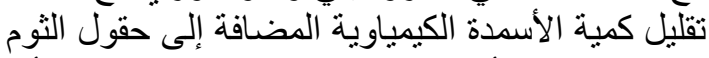

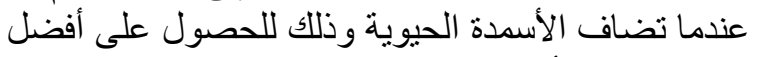

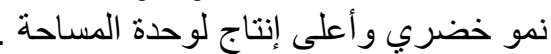

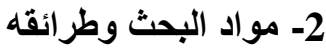

نفذت الدراسة في حقل الخضر اوات ات التابع لقسم البستنة

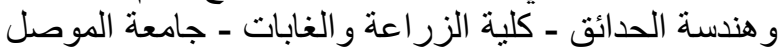

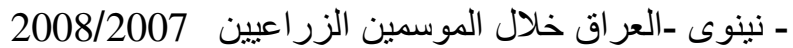

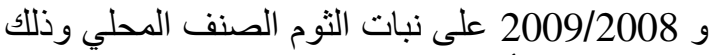

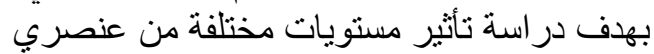
النتروجين و الفوسفور و السماد الحيوي ميتي - النتروبين (سماد

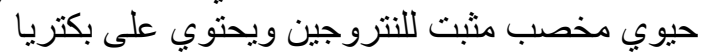
الازوتوباكتر المثبتة للنتروجين ، من إنتاج الهيئة العامية العزية

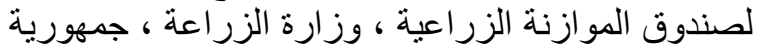

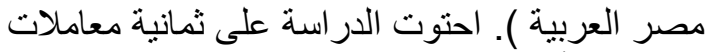

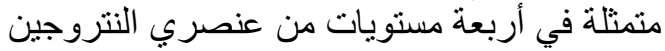

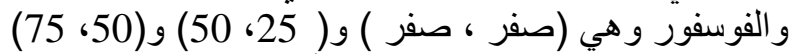
و(75، 150 ) بالإضافة إلى التلقيح أو عدم التلقيح بسماد النتروبين ـ اجري تحليل لتربة الحقل حسب مالأل هو مثبت في

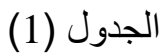
زرعت فصوص الثوم الصنف المحلي في 2008/9/20/20 و 2008/9/15 على مروز بطول 3 متر والمسافة بين

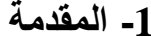

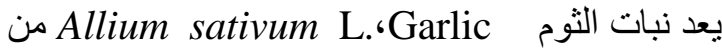

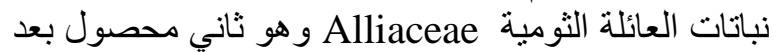

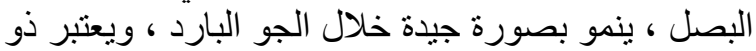

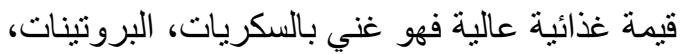

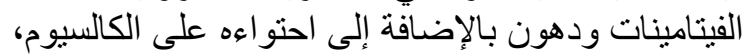

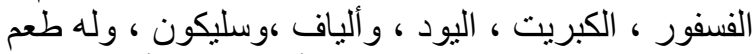

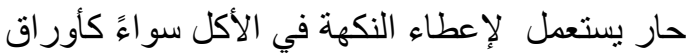

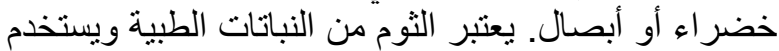

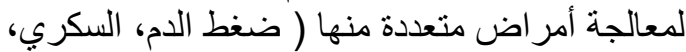
أمر اض الروماتزم، وكذلك التقرحات) Xiaohongx and . Masahiko (2002)

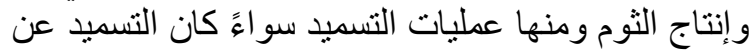

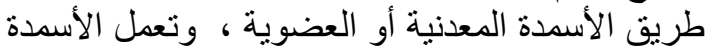

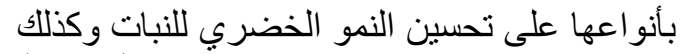

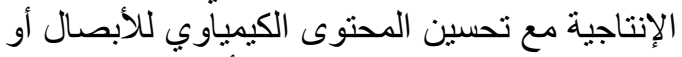

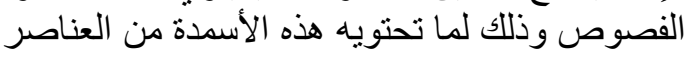

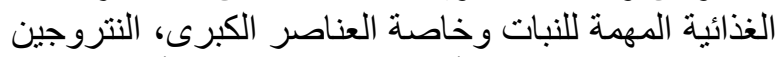

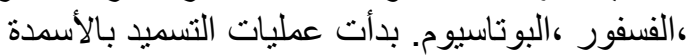

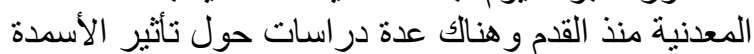

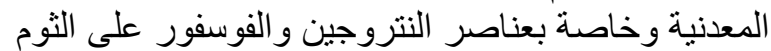

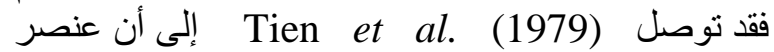
النتروجين أدى إلى زيادة معنوية في صنفات النمو الخضى النضري

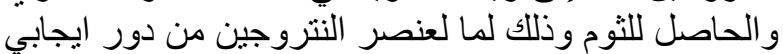

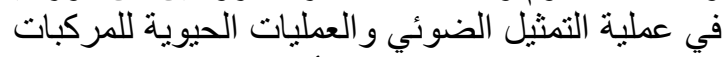

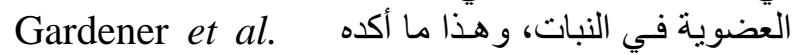

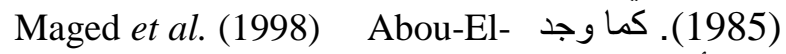

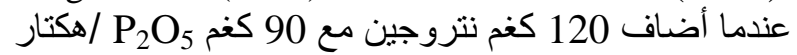
حيث لاحظ أن هناك زيادة معنوية في الحاصل الكلي ـ أما El-Seifi et al. (2004)

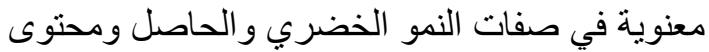

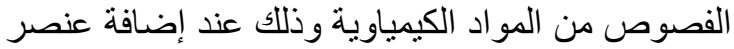

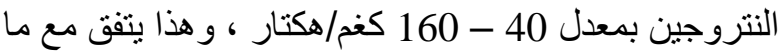
توصل إليه Tomas and Kielian وكنمالك Silvia and Lipiksi (2008) get al. (2007) إضافة عنصر النتروجين و الفوسفور أدى إلى حدوث زيادة

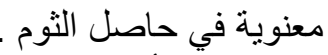
وتعد الأسمدة الحيوية من أهم التقنيات التي تستخدم في

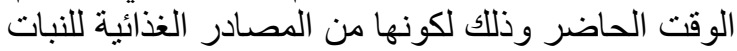

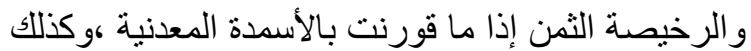

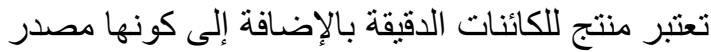

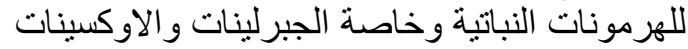

Haller and Stople (1985) تم التوسع فى استخدام هذه الأسمدة بسبب قدرتها على التى

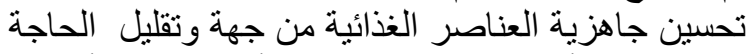

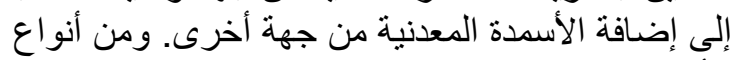

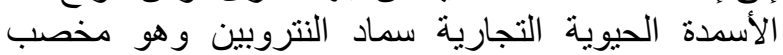




\begin{tabular}{|c|c|c|c|}
\hline \multicolumn{4}{|c|}{ جدول(1): بعض الصفات الكيميائية والفيزيائية لتربة حقل الاراسة ** } \\
\hline التقاير & مكونات التربة الفيزيائية الفية & التقدير & الصفات الكيميائية \\
\hline 43,47 & Sand رمل Sa & 6,2 & درجة حموضة التربة pH \\
\hline 42,36 & Silt \% غرين & 19,78 & المادة العضوية O.M غ/كغ \\
\hline 15,16 & Clay \% طين & 931 & النتروجين جزء بالمليون \\
\hline تربة لومية & نexture ن نسجة التربة & 8,42 & الفوسفور جزء بالمليون \\
\hline & & 80,74 & البو تاسيوم جزء بالمليون \\
\hline
\end{tabular}

*حلت العينات في قبم المختبرات والاراسات التطبيقية ، مديرية زراعة نينوى، وزارة الزراعة ،العراق.

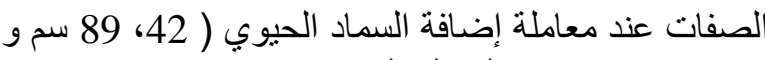

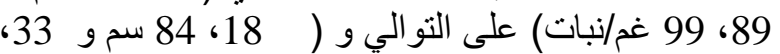

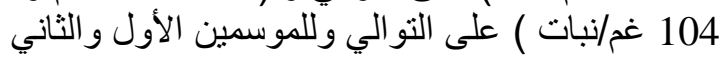

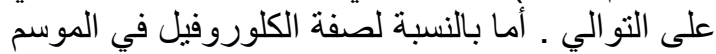

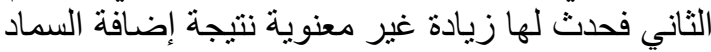

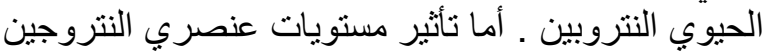

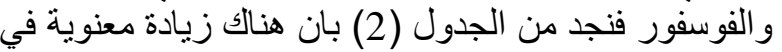

صفة طول أطول ورقة وكذللك الحاصل البيولوجي ولكنان الموسمين نتيجة لإضافة عنصري وكري النتروجين و الفوسفور

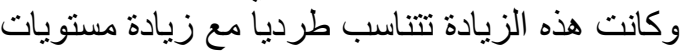

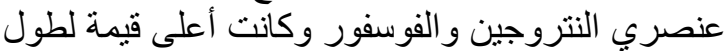
أطول ورقة 87، 90 سم في الموسم الأول و وعند المستوى ألوى

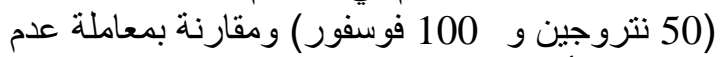
الإضافة ، أما في الموسم الثاني فكانت

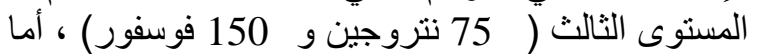
الحاصل البيولوجي فكان 69، 110 و و 94، 94، 109 غم /نبات

وللموسمين الأول والثاني على التو الي وذلك الك عند معاملة

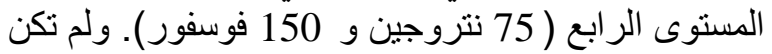

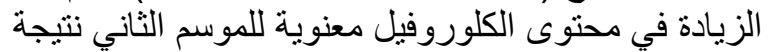

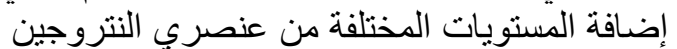

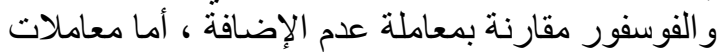

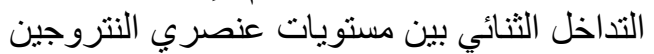

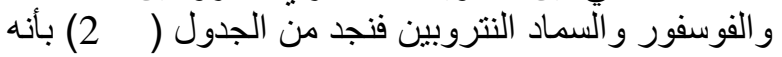
هناك فروقات معنوية بين اغلب معاملات التداخل الثنائي و ان معاملة النداخل الثنائي بين المستوى ( 50 و 100 ) ) من عنصري النتروجين والفوسفور مع إضافة الفئة السماد الحيوي النتروبين أدت إلى إنتاج أعلى طول للتوبن اللورقة

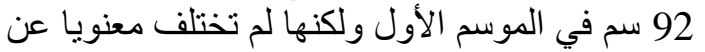

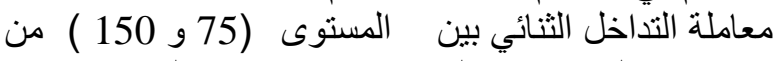
عنصري النتروجين و الفوسفور ومع إضافة النتروبين. وقد الند

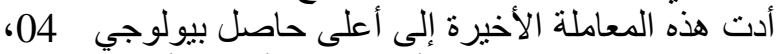
120 غم/نبات في الموسم الأول وكذلإلك أعطت أعلى أعلى طول

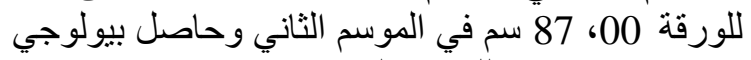

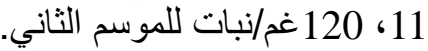

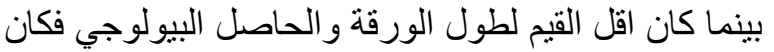

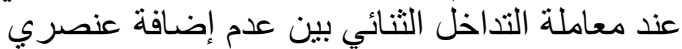

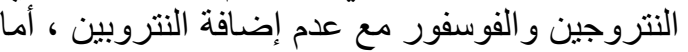
بالنسبة لمحتوى الكلوروفيل في الأوراق في الموسم الثاني

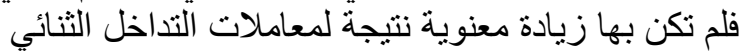
مقارنة بمعاملة المقارنة . مادية
المروز 75 سم و المسافة بين الفصوص ضمن المرز 10

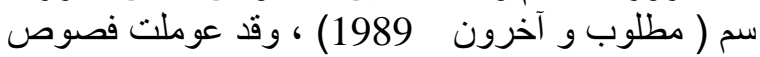
الثوم بالتلقيح بالسماد الحيوي قبل الزر اعة اعل بالمخصب

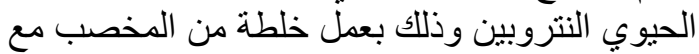

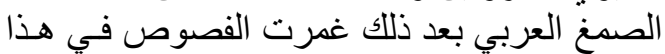
المخصب لمدة 5-3 دقائق ( (El- Seifi et al., 2004)

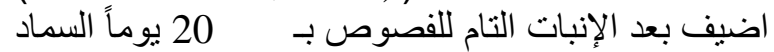

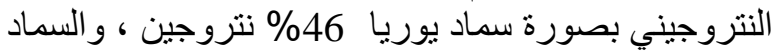
الفوسفوري بصورة

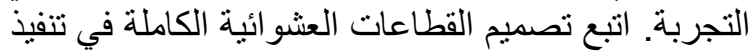
التجربة وبثلاثة مكرر ات و وأجريت كافة عملية التيات الخدمة

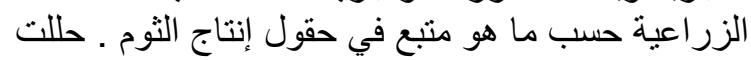

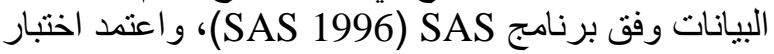

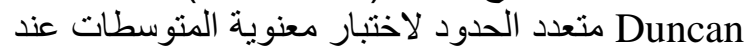

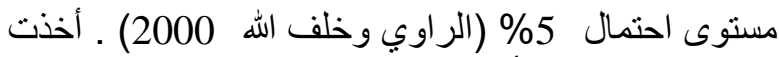

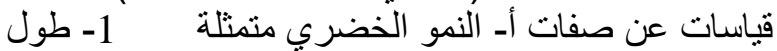
أطول ورقة (سم) و 2- نسبة الكلوروفيل الكلي في الأور اق بن

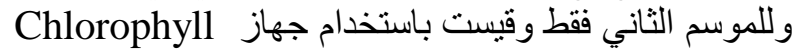
Meter SPAD 502

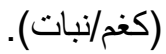

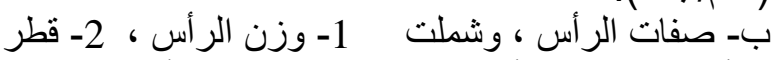

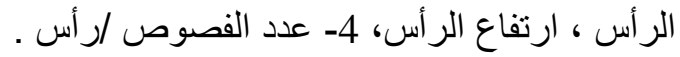

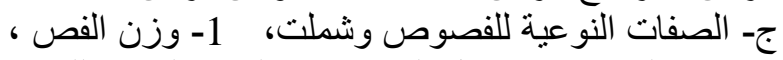

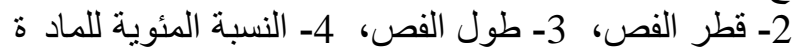

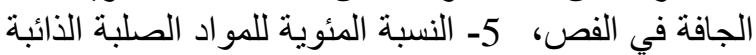
Hand Refract و التي تم تقدير ها بجهاز TSS meter د- الإنتاج الكلي لوحدة المساحة (طن/هكتار).

\section{3- النتائج والمناقشة}

1-3-3 صفات النمو الخضري

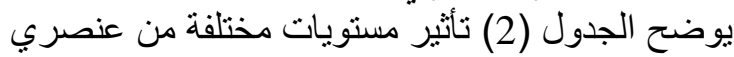

النتروجين و الفوسفور و السماد الحيوي النترئي

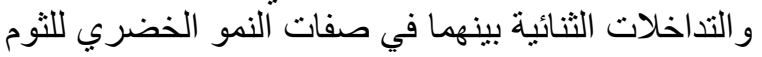

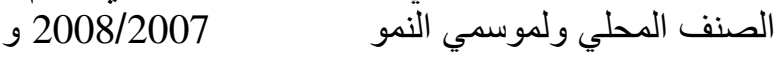
2009/2008 ـ ويظهر من الجدول بان إضافة السماد

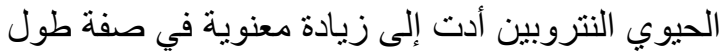

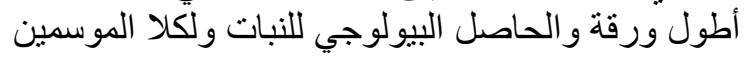

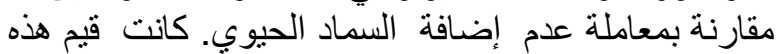


جدول (2): تأثير التسميد المعدني (النتروجيني والفوسفوري) والسماد الحيوي النتروبين في صفات النمو الخضري للثوم (الصنف المحلي) لموسمي النمو 2008/2007 * $2009 / 2008$ و

\begin{tabular}{|c|c|c|c|c|c|c|c|}
\hline \multicolumn{3}{|c|}{$2009 / 2008$} & \multicolumn{2}{|c|}{$2008 / 2007$} & \multicolumn{3}{|c|}{ المعاملات السمادية } \\
\hline الكتي في الأوراق & الحاصل البيولوجي & طول أطول ورقة (سم) & (غاصل البيولوجي & طول أطول ورقة & النتروبين & $\mathbf{P}$ & $\mathbf{N}$ \\
\hline 163,89 & 73,69 & ج,92,92 & ج 65,83 & د 66,91 & - & صفر & صفر \\
\hline 64,18 & ا & إ,53,53 & 年 & ( 85,29 & + & صفر & صفر \\
\hline 63,89 & ا & 年, 92 & 吾 & 26, 26 79 & - & 50 & 25 \\
\hline 64,44 & 1116,33 & أ 50, & 20 20 & I 89,64 & + & 50 & 25 \\
\hline 62,26 & 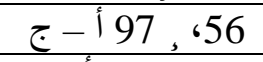 & ب 78,33 & 99,47 أب & 17, 89 أب & - & 100 & 50 \\
\hline 65,48 & 101,11 أب & 186,67 & 119,03 & $\mid 92,57$ & + & 100 & 50 \\
\hline 65,72 & 76, 99 أب & أ 42, 83 أب & 101,34 أب & ج 85,85 & - & 150 & 75 \\
\hline 62,02 & أ 120,11 & أ 87,00 & 120,04 & 190,16 & + & 150 & 75 \\
\hline 63,94 & ب8,03 88 & 4, 40, 77 ب & ب8,37 88 & 30,30 & - & \multirow{2}{*}{\multicolumn{2}{|c|}{ التأثيّر العام للنتروبين }} \\
\hline I 64,03 & أ 104,33 & 184,18 & 199,89 & 189,42 & + & & \\
\hline أ 64,06 & 74,74 76 ج & 73, 73 ب & 57, 73 ج & 10, 67 ج & صفر صفر & \multirow{4}{*}{\multicolumn{2}{|c|}{ للمعاملات التمأثير العادية }} \\
\hline I 64,17 & 72, 98 ب & أ 71, 81 أب & 83,03 ب & 84,45 & 50 & & \\
\hline I 63,87 & 34, 99 أ ب & 182,50 & I 109,25 & 190,87 & 100 & & \\
\hline 63,87 & أ 109,94 & أ 85,21 & ,69, 69 أ 110 & 00, 84 ب & 150 & & \\
\hline
\end{tabular}

* المعدلات المشتركة بنفس الحرف الأبجدي لكل عامل على حدى و التداخل بينهما لا تختلف معنويا حسب اختبار Duncan المتعدد الحدود وعند مستوى احتمال 5\%. 
90، 4 سم قطر الرأس و 20، 33 عدد الفصوص بالر أس أس الف مقارنة بمعاملة عدم إضافة و التي أعطت اقل القديم القيم لصفات

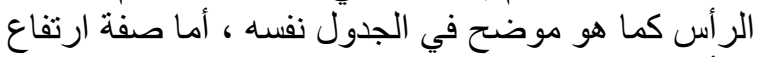

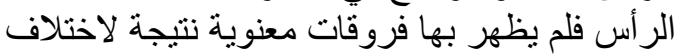
المعاملات السمادية. وكان لمعاملات التداخل الثنات لثنائي بين

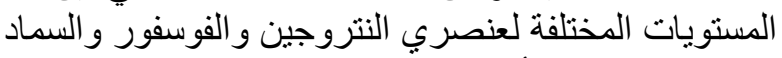

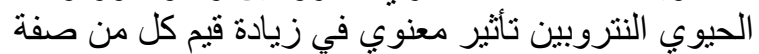

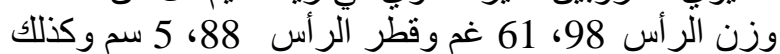
ارتفاع الرأس 40، 5 سم للموسم الأول نتيجة لمعاملة

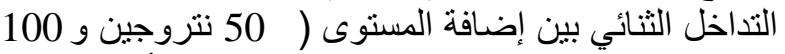
فوسفور ) ومع إضافة السماد الحيوي النتروبين ،أما صفية عدد الفصوص وص بالر أس فكان 34، 49 نتيجة لمعاملة التداخل

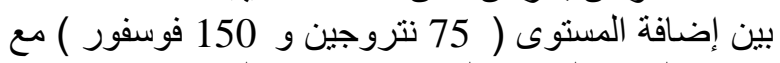

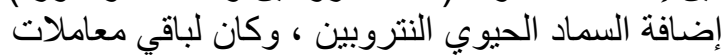
الأنداخل الأخرى اختلافات معنوية في صفة الر أس للموسم

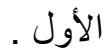

أما في الموسم الثاني فقد تمبزت معاملة التداخل بين

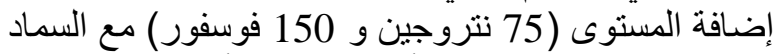

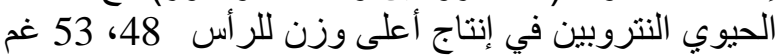

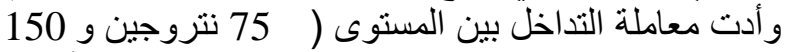
فوسفور ) وبدون إضافة السماد الحيوي التنتروبين إلى أعلى

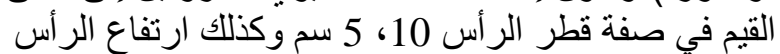

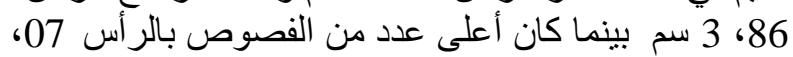

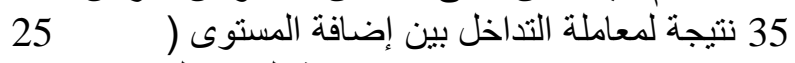
نتروجين و 50 فوسفور ) مع إضافة السماد الحيوي لئل

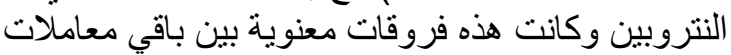

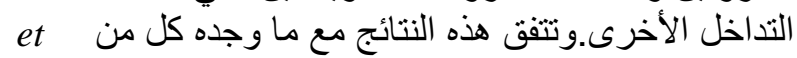
El- El- Shabasi et al.,2003 و Ali al., 2001 2004b Bardisi et al., gSeifi et al.,2004 إضافة السماد المعدني النتروجيني و الفوسفوري مع السماد التئي

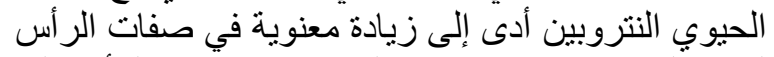

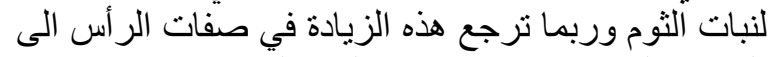

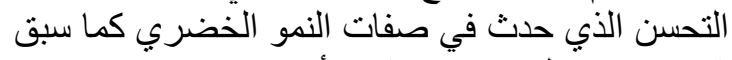

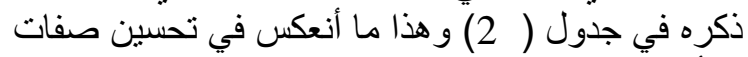

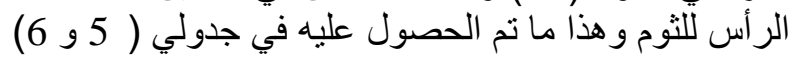

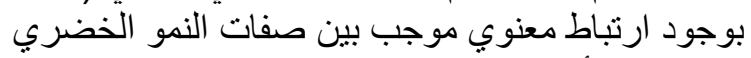
وصفات الرأس فقد كانت قيم الارتباط بين طول الورت الورقة وكل

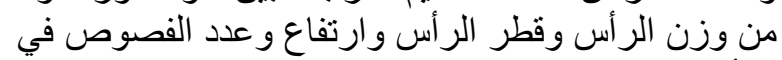

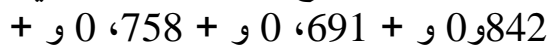
الرأس (+ الرن 852، 0 على التو الي وللموسم الأول (2008/2007 ) ، أما لـ الما

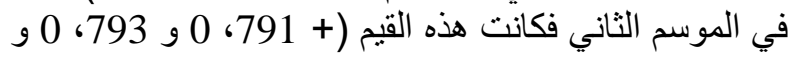

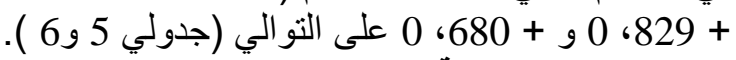
3-3-3 الصفات النوعية للفص يوضح جدول (4) نأثير مستويات مختلفة من عنصري النتروجين و الفوسفور والسماد الحيوي النتروبين في سئري

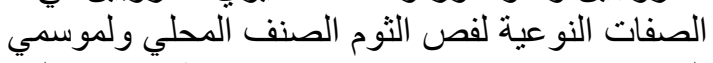

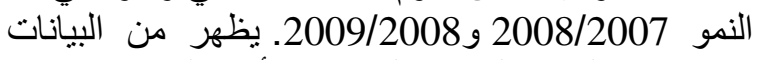
بان إضافة السماد الحيوي النتروبين أدت إلى زيادة معنوية
وتيتق هذه النتائج مسع ما توصل إليه كل من - Abou

El-Seifi et al.,2004 El-Maged et al.,1998

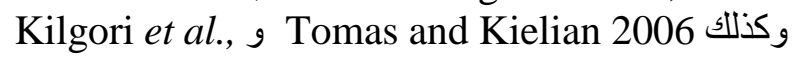
2007 من انه حدثت زيادة معنوية وتحسين في صفات

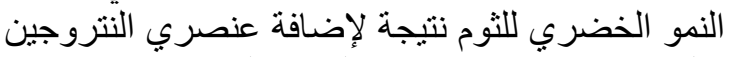

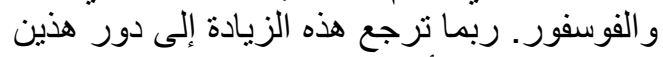

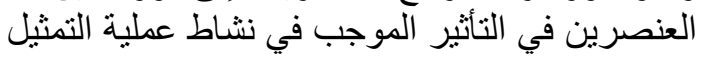
الضوئي و العمليات الحيوية للمركبات العضوية في النبات النيات Gardener et al., 1985

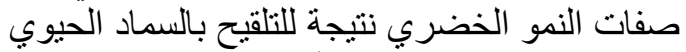

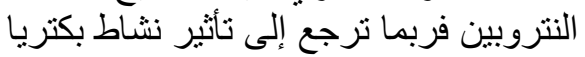
Azotobacter

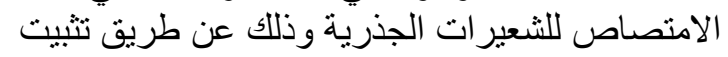

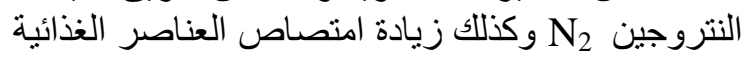

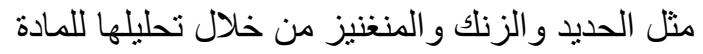
العضوية في التربة وبالتالي توفر هذه العناصر بصورة Bardisi et و Bhonde et al., 1997 قابلة للامنصاص . al., 2004a 2-3-3 صفات الرأس

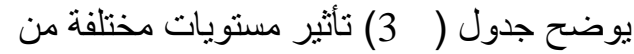
عنصري النتروجين والفوسفور والسماد الحيوي النتروبين

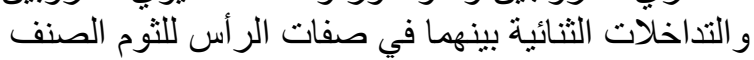

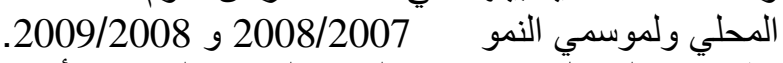
يظزهر من الجدولَ بان إضافة السماد الحيوي النتروبين أدت

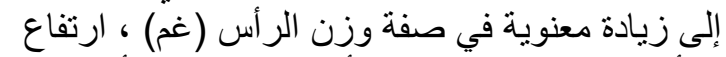

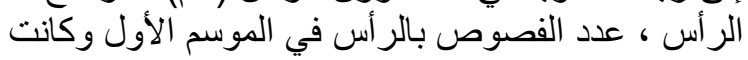

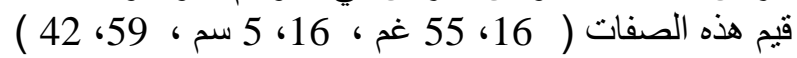

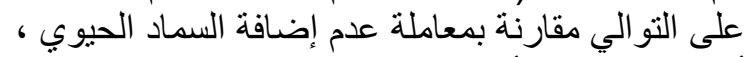

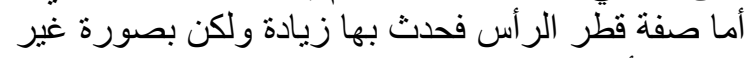

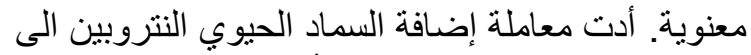

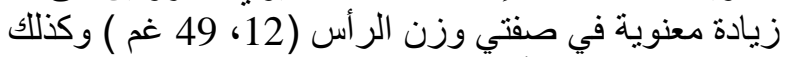
عدد الفصوص بالر أس 79 79، 32 في الموسم الثاني مقارنة

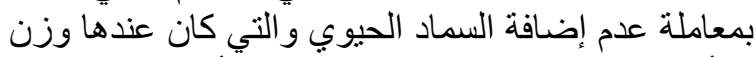

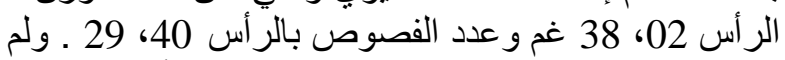
تكن الزيادة معنوية في صفتي قطر و ارتفاع الر أس للموسم

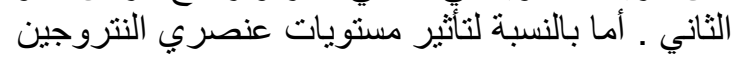
و الفوسفور فتوضح النتائج في الجدول ( 3 3) بان معاملات

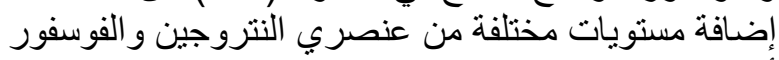

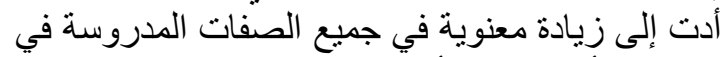

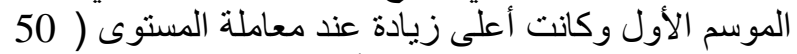

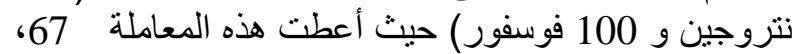

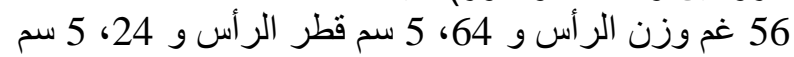
ارتفاع الر أس مقارنة بمعاملة عدم الإضافة بـ بينما أدت معاملة

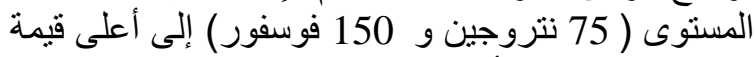

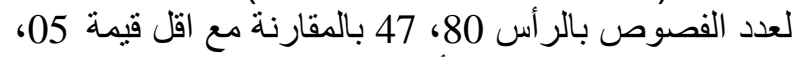
34 عند معاملة الإضافة ـ أما في الموسم الثاني فكان 75

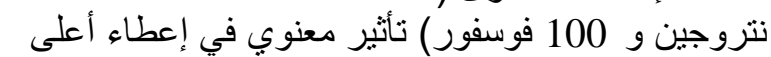

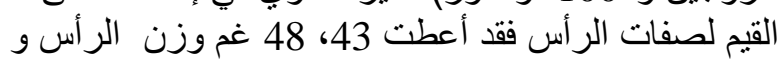


جدول (3): تأثير التسميد المعدني (النتروجيني و الفوسفوري ) والسماد الحيوي النتروبين في صفات الرأس للثوم (الصنف المحلى) ولموسمي النمو 2008/2007 و2009/2008/

\begin{tabular}{|c|c|c|c|c|c|c|c|c|c|c|}
\hline \multicolumn{4}{|c|}{$2009 / 2008$} & \multicolumn{4}{|c|}{$2008 / 2007$} & \multicolumn{3}{|c|}{ المعاملات السمادية } \\
\hline عدال الفصوص & 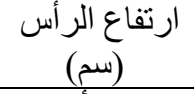 & 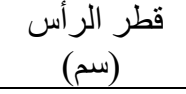 & وزن الرأس & عدد الفصوص & $\begin{array}{c}\text { ارتفاع الر أسم) } \\
\text { أس }\end{array}$ & 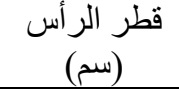 & وزن الرأس (غر) & النتروبين & $\mathrm{P}$ & $\mathrm{N}$ \\
\hline ب 24,53 & (3,57 & 4,4,49 ب ج & 31,02 & ه 31,4 & 3,30 & 4,90 4 ج & د 38,95 & - & صفر & صفر \\
\hline 07, 28 أ ب & 69,69 أ ب & أ 63, 4 أب & 35,35 أـ د 42, & 36,70 & 4,87 ج & د,03 & 49,96 ب & + & صفر & صفر \\
\hline 40, 29 أب & 41, 31 ب ج & 47, 47 ب ج & 13, 38 ب ج & 58, 40 ج د & د, 40 & 19, 5 ب ج & 14, 45 ج د & - & 50 & 25 \\
\hline 135,07 & 13,72 & 15,07 & 25,05, 53 أب & 41,55 & 10, 5 أب ب & ب, 28, & ب5,87 & + & 50 & 25 \\
\hline 20, 31 أب & 28, 28 & 4,09 ج & 55, 39 ب -د & 15, 44 ب ج & 5,07 أ- ج & 5,39 & 36, 31, ب ج & - & 100 & 50 \\
\hline I 34,07 & 13,82 & 88, 4 أ ب & ( 47,60 & 78,78 ب ج & 15,40 & $\mid 5,88$ & | 61,98 & + & 100 & 50 \\
\hline 32,47 أ ب & 13,86 & $\mid 5,10$ & د 43,37 & 25, 46 أب & 07,07 أ- ج & (22, 5 ج & 86, 50 ب ج & - & 150 & 75 \\
\hline I 33,93 & (3,49, أ- & 4,70 أب & 153,48 & 149,34 & 15,27 & 6,83 & 54,81 & + & 150 & 75 \\
\hline 40, 29 ب & 13,53 & 14,54 & 02,02 & 60، 40 ب & (54,54 & 5,18 & 58, 56 ب ب & - & ل للنتروبين & التأثنير ال \\
\hline 132,79 & 13,68 & 14,82 & i 49,12 & 142,59 & 15,16 & 15,26 & 55,16 & + & & \\
\hline (26,30 & 13,63 & 4,56 ب ب & 69، 36 ج & 05، 34 ج & 4,09 & 4,47 & 46,44 ب & صفر صفر & العام & \\
\hline 132,24 & 13,56 & 77, 4 أ ب & 59, 45 أب & 41,07 ب & 90, 40 & 15,24 & 51, 59 ب & 50 & السمادية & للمعاملا \\
\hline I 32,64 & 13,55 & 4,49 ب & 58,53 ب ب & 48, 43 ب & $\lcm{5,24}$ & 15,64 & 156,67 & 100 & & \\
\hline I 33,20 & 13,68 & 14,90 & 48,43 & 47,80 & $\lcm{5,17}$ & 15,53 & I 52,84 & 150 & & \\
\hline
\end{tabular}


جدول (4): تأثير التسميد المعدني (النتروجيني والفوسفوري) والسماد الحيوي النتروبين في صفات النوعية لفصوص للثوم (الصنف المحل) ولموسمي النمو 2007/ * 2009 م 2008 و 2008

\begin{tabular}{|c|c|c|c|c|c|c|c|c|c|c|c|c|}
\hline \multirow{2}{*}{\multicolumn{5}{|c|}{$2009 / 2008$}} & \multirow{2}{*}{\multicolumn{5}{|c|}{$2008 / 2007$}} & \multirow{2}{*}{\multicolumn{3}{|c|}{ المعاملات السمادية }} \\
\hline & & & & & & & & & & & & \\
\hline للمادة الجافية النئة & TSS & قطر الفصم) & $\begin{array}{c}\text { طول الفص) } \\
\text { (سم) }\end{array}$ & وزن الفص & 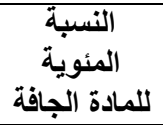 & TSS & قطر الفصم & $\begin{array}{c}\text { طول الفص) } \\
\text { (سم) }\end{array}$ & وزن الفص & النتروبين & $\mathbf{P}$ & $\mathbf{N}$ \\
\hline 00, 44 ب & 16,82 ج & 94,94 0 ج & 49, 29 & i 1,99 & د39,65 & 47, 17 د & 16 16 16 ج & (92 1 ج ج & 88 1 88 & - & صفر & صفر \\
\hline 06, 46 أب & (19,77 أ- ج & 20, 1 أب & 83, 2 أب & 90, 1 أب & 10, 43 ج & 80, 20 ب ج & 24، 1 ب ج & (96 1 96 ج & 07، 2 ب ج & + & صفر & صفر \\
\hline 44 ب1 & ج 19 83 & 40, 1 أب & ب 2,77 & 93, 1 أب & 25, 44 ب ج & 20,13 جد & 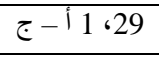 & 47، 2 & 23، 2 أب & - & 50 & 25 \\
\hline 83, 45 أب & 83, 21 أب & 1, 19 أب & 85, 2 أب & 12,16 & 09, 46 أ ب & 124,73 & 26، 1 ب ج & 45 25 & 15، أ 25 & + & 50 & 25 \\
\hline 20، 45 أب & 22, 20 أب & 1, 1 1 أب & 12,97 & 1,43 ب & 50, 44 ب ج & 03, 23 أ- ج & 42، 1 أب & 90، 2 أب & 23، 2 أب & - & 100 & 50 \\
\hline 146,56 & 122,90 & 11,23 & 13,03 & 12,16 & I 47,48 & 50,51 21 أ- ج & 33، 1 أ -- ج & 12 ، 36 & I 2 ، 41 & + & 100 & \\
\hline 90, 45 أب & 77, 19 أ - ج & 1,19 أب & 12,97 & 12,37 & 48, 43 ج & 67, 23 أ ب & 36، 1 أب & 13616 & 37، 2 أب & - & 150 & 75 \\
\hline I 47,15 & 13, 19 ج & 1,07 ب ب & 2,87 أب & 12,05 & 47,52 & 47, 20 ب -د & i 1 ، 47 & 13628 & 29، 2 أب & + & 150 & 75 \\
\hline ب 44,78 & 19,16 & 11,18 & 12,80 & 1,93 ب & (97, 92, ب2 & I 21,08 & ín 11 31 & أ 61 2 أ & $12 ، 18$ & - & \multirow{2}{*}{\multicolumn{2}{|c|}{ التأثير العام للنتروبين }} \\
\hline $\mathbf{1} 46,40$ & I 20,91 & $\{1,18$ & 12,90 & $\lceil 2,07$ & أ 46,05 & $\mid 21,88$ & ín 1 33 & $12 ، 51$ & ín 23 & + & & \\
\hline 03, 45 أب & 18 ب ب & 1,07 ب & 12,66 & 95, 1 ب & 31, 41 ب & 14, 19 ب & I 1 ، 20 & 94، 1 ج & 98 98، 1 ب & صفر صفر & \multirow{4}{*}{\multicolumn{2}{|c|}{ للمعاملات السمادية التأثير }} \\
\hline 92, 44 أ ب & I 20,83 & 11,30 & 12,81 & 12,05 & 17, 45 أ ب & 22,43 & I 1 ، 28 & 46، 2 ب & I 19 & $50 \quad 25$ & & \\
\hline 88, 45 أب & $\mid 21,56$ & 11,20 & 13,00 & ب 1,80 & 145,99 & $\mid 22,27$ & ín 1 .38 & 63، 2 ب3 & 12.32 & 100 & & \\
\hline 146,53 & 19,45 & 11,13 & 12,92 & 12,21 & I 45,50 & 22,07 & أ 42 1 أ & $13 ، 22$ & $12 ، 33$ & 150 & & \\
\hline
\end{tabular}




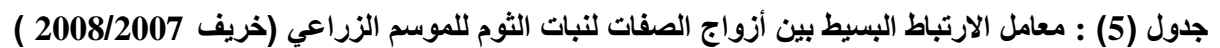

\begin{tabular}{|c|c|c|c|c|c|c|c|c|c|c|c|}
\hline (الإنتاج الكلئ & النسبة المئوية للمادة & TSS & قطر الفصم & طول الفص (سم) & وزن الفص & عداد الفصوص & 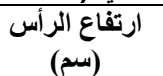 & قطر الرأسم) & وزن الرأس & حاصل البيولوجي & الصفات \\
\hline \multirow{11}{*}{$* 0,504$} & $* 0,524$ & $* 0,423$ & $* * 0,775$ & 0 ،351 & $* * 0,763$ & $* * 0,852$ & $* * 0,758$ & $* * 0$ ،691 & $* * 0,842$ & $* 0$ ، 439 & طول أطول ورقة (سم) \\
\hline & $* * 0,638$ & * 0,558 & $* \mathbf{0}, \mathbf{5 5 0}$ & $* 0,569$ & $* 0,498$ & $* * 0,661$ & $* 0,582$ & $* * 0 ; 607$ & $* 0,412$ & * 0 ، 410 & الإتتاج الكلى (طن/هكتار) \\
\hline & & 0,264 & * 0,444 & * 0,433 & $\mathbf{0 , 3 3 2}$ & $* 0,540$ & * 0,487 & * 0 ، 471 & $* 0,431$ & 0 ، 373 & الجافبة المئوية للمادة \\
\hline & & & $\mathbf{0}, \mathbf{3 3 0}$ & 0,317 & * $\mathbf{0}, 535$ & 0,346 & * $\mathbf{0}, \mathbf{5 2 0}$ & $* 0$ ×514 & 0,267 & $\mathbf{0}, 335$ & TSS \\
\hline & & & & & * 0,563 & $* * 0,613$ & $* 0,572$ & *80,651 & $* * 0,642$ & 0,174 & قطر الفص (سم) \\
\hline & & & & & 0,382 & $* 0,530$ & 0,385 & $* 0,493$ & $\mathbf{0}, 371$ & 0,155 & طول الفص (سم) \\
\hline & & & & & & $* * 0,740$ & $* * 0,772$ & $* * 0,812$ & $* * 0,668$ & 0,278 & وزن الفص (غم) \\
\hline & & & & & & & $* * 0,814$ & $* * 0,672$ & $* * 0,866$ & 0,204 & عدد الفصوص /رأس \\
\hline & & & & & & & & $* * 0,730$ & $* * 0,857$ & $* 0,484$ & ارتفاع الرأس (سم) \\
\hline & & & & & & & & & $* * 0,617$ & 0,334 & قطر الرأس (سم) \\
\hline & & & & & & & & & & 0,347 & وزن الرأس \\
\hline
\end{tabular}

جدول (6) : معامل الارتباط البسيط بين أزواج الصفات لنبات الثوم للموسم الزراعي (خريف2009/2008 )

\begin{tabular}{|c|c|c|c|c|c|c|c|c|c|c|c|}
\hline الإنتاج الكلي & النسبة المئوية للمادة & TSS & قطر الفص) & طول الفصم & وزن الفص) & عدد الفصوص أس & $\begin{array}{c}\text { ارتفاع الرأس) } \\
\text { (سم) }\end{array}$ & قطر الرأس & وزن الرأس & حاصل البيولوجي & الصفات \\
\hline \multirow[t]{12}{*}{$* * 0,631$} & $*_{0.443}$ & $* * 0,612$ & 0,388 & $* * 0,618$ & $* * 0.672$ & $* * 0$; 680 & $* * 0829$ & $* * 0,793$ & $* * 0.791$ & $* 0511$ & طول أطول ورقة (سم) \\
\hline & $* 0,511$ & $* 0,494$ & $* 0,452$ & $* * 0,675$ & $* 0,419$ & $* 0,547$ & $* 0,506$ & $* 0,568$ & $* 0,562$ & $* 0,401$ & الإنتاج الكلي (طن/هكتار) \\
\hline & & 0,211 & $* * 0,600$ & $* * 0,764$ & * 0,572 & $* 0,552$ & * 0,555 & $* 0,504$ & * 0,504 & $* * 0,663$ & الجافبة المئوية للمادة \\
\hline & & & 0,218 & $* * 0,611$ & $* 0,548$ & $* 0,521$ & $* 0,579$ & $* \mathbf{0}, \mathbf{5 0 0}$ & $* 0,499$ & $* * 0,632$ & TSS \\
\hline & & & & $* 0,418$ & $* 0,402$ & 0,303 & 0,377 & 0,355 & $\mathbf{0}, 351$ & 0,298 & قطر الفص (سم) \\
\hline & & & & & $* 0,543$ & $* * \mathbf{0}, 950$ & $* * 0,731$ & $* * 0,747$ & $* * 0,746$ & 0,268 & طول الفص (سم) \\
\hline & & & & & & $* * \mathbf{0}, 717$ & $* * 0,862$ & $* * 0,846$ & $* * 0,851$ & $\mathbf{0 , 3 7 7}$ & وزن الفص (غم) \\
\hline & & & & & & & $* * 0,865$ & $* * 0,854$ & $\begin{array}{r}\mathbf{0} \cdot 800 \\
* *\end{array}$ & $* * 0,876$ & عدد الفصوص /رأس \\
\hline & & & & & & & & $* * 06907$ & $\begin{array}{r}0 \text { ،971 } \\
* * *\end{array}$ & $* * 0$ ، 865 & ارتفاع الرأس (سم) \\
\hline & & & & & & & & & $\begin{array}{r}1 \text { (000 } \\
* *\end{array}$ & $* 0 ، 445$ & قطر الرأس (سم) \\
\hline & & & & & & & & & & * 0 ، 442 & وزن الرأس \\
\hline & & & & & & & & & & 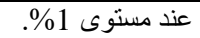 & * عند مستوى 5\%. \\
\hline
\end{tabular}



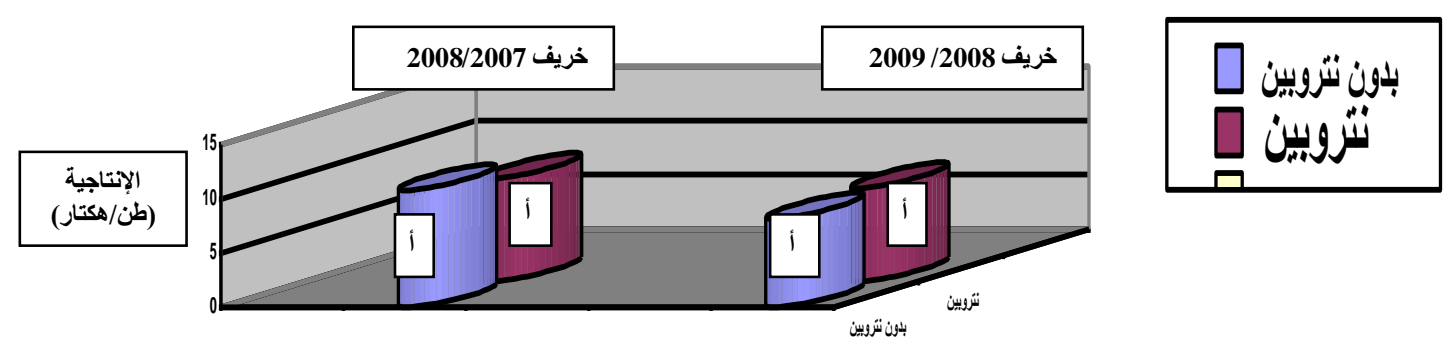

شكل (1) : تأثير السماد الحيوي النتروبين في إنتاجية الثوم لوحدة المساحة لموسمي الزراعة ( خريف2008/2007 و2008/2009) :

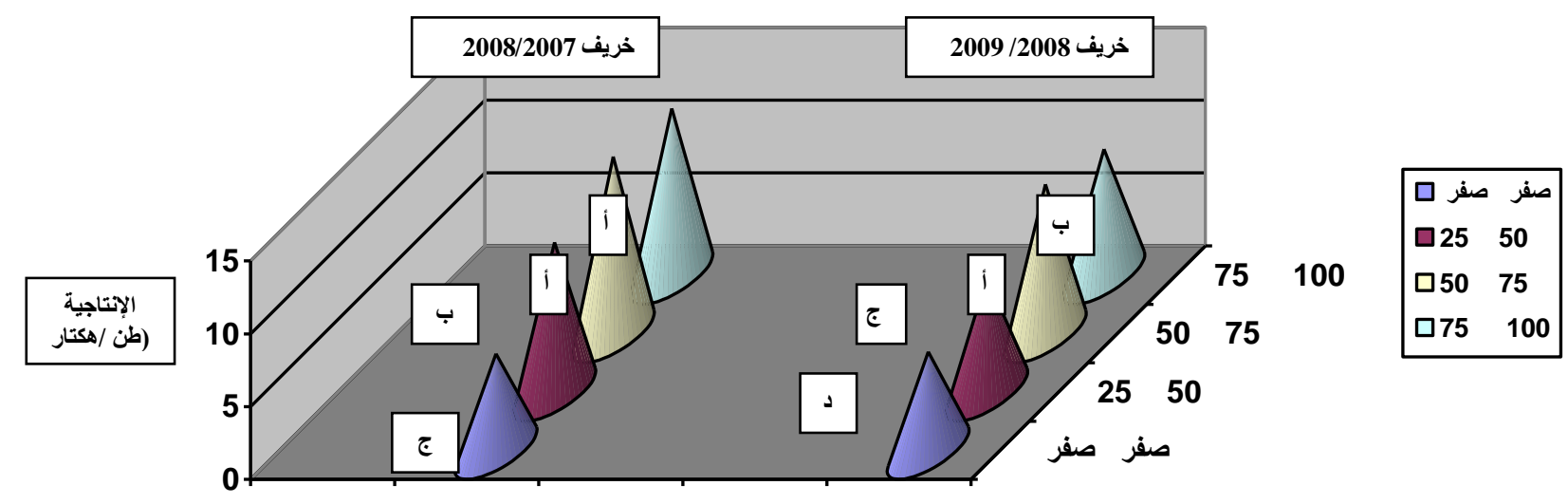

شكل(2): تأثير التسميد المعدني (النتروجيني و الفوسفوري ) في إنتاجية الثوم لوحدة المساحة لموسمي الزراعة (خريف . 2008/2007 و 2009/2008) 


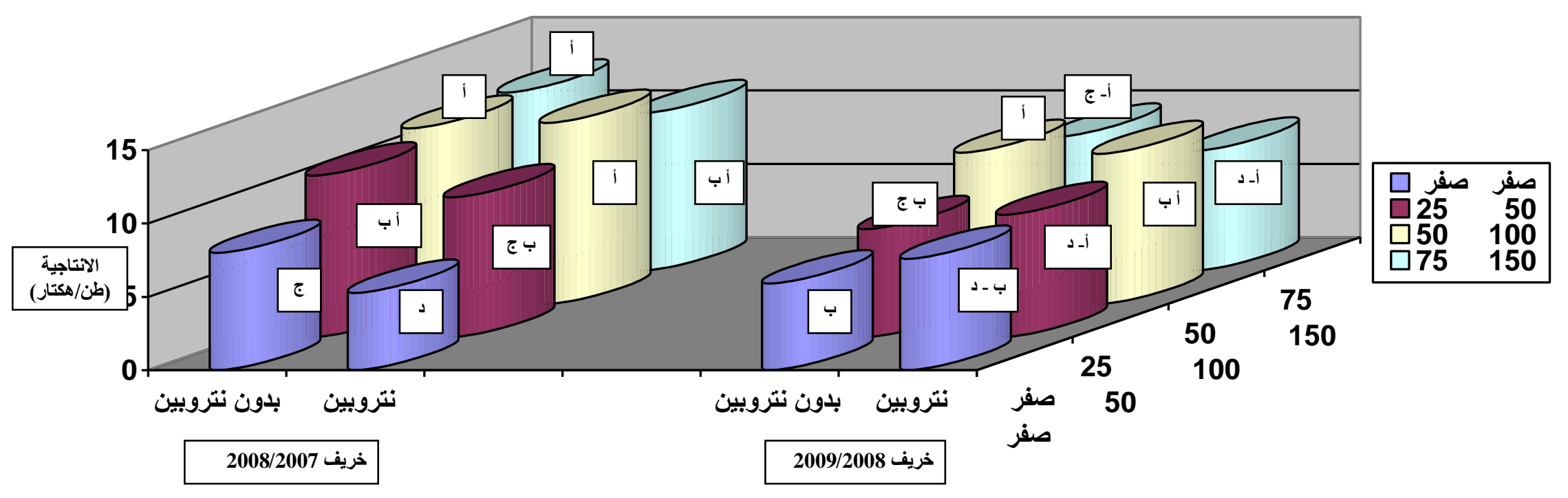

شكل (3) : تأثير التداخل بين التسميد المعني (النتروجيني والفوسفوري ) والسماد الحيوي النتروبين في الإتتاجية لوحدة المساحة لنبات الثوم ولموسي الزراعة ( خريف ) 2008/2007 و 2008 /2009 ). 
طن/هكتار للموسمين الأول و الثناني على التو الي نتيجة

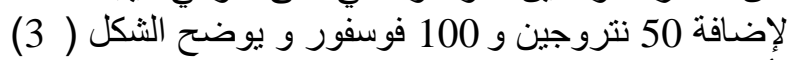
تأثثير التداخل بين مسنويات عنصر النتروجين و الفوسفور و السماد الحيوي النتروبين وان ألتاني أعلى إنتاجية لوحدة المساحة كانت 32، 12 طن/هكتار للموسم الأول عند معاملة التبن التداخل

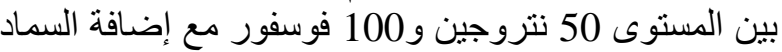
الحيوي النتروبين أما في الموسم الثناني فكانت الإنتاجية 28،

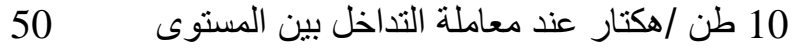

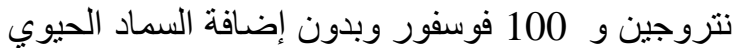
ولكنها لم تختلف معنويا عن معاملة التداخل بين المستويات

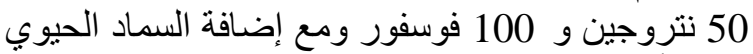

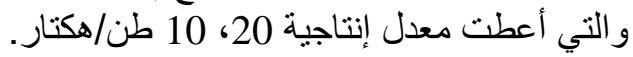

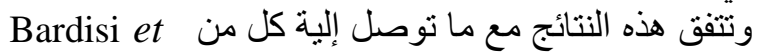

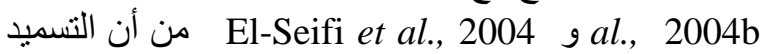
بكل من عنصري النتروجين و الفوسفور وكذللك التسميد

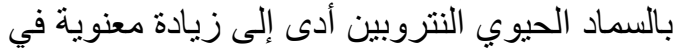

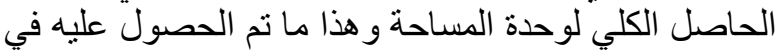

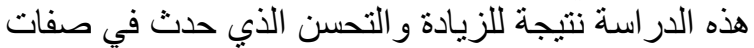

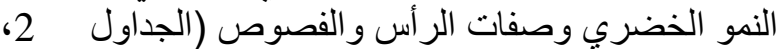

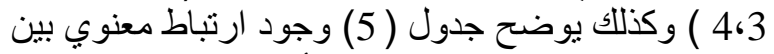

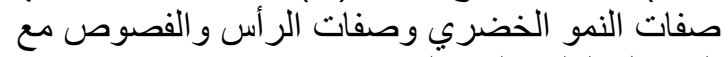
الحاصل الكلي ولكلا ألموسمين .

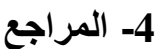

مطلوب، عدنان ناصر وكريم صالح عبدول و عز الدين الدين

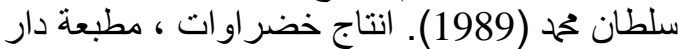

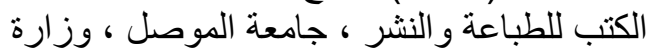

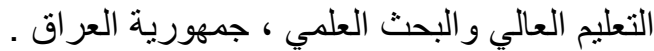
الراوي، خاشع محمود و عبد العزيز خلف الله ( 2000). تصميم وتحليل التجارب الزر اعية (الطبعة الثانية)،

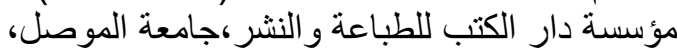

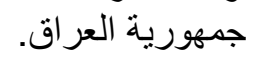

Abou El- Maged M. M., Abdalla A.M and Yousef R.A.(1998). Response of garlic growth, chemical content, bulb yield and quality to N,P or $\mathrm{K}$ fertilization .Egypt. J. Appl. Sci. 13(6): 151-174.

Ali A. H. , Abdel Moaty M.M and Shaheen A.M. (2001). Effect of bionitrogen, organic and inorganic fertilizer on the productivity of garlic (Allium sativum L.) Plants .Egypt J. Appl. Sci.16(3): 173-188.

Bardisi A., El-Mansi A.A. , Fayad A.N. and Abou El-Khair E.E. (2004 a). Effect of mineral NP and Boifertilizers on garlic under sandy soil conditions A- Growth
في كل من وزن الفص 233، 23، 2 غم وكذللك قطر الفص

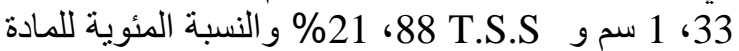

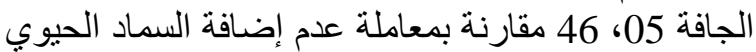
وللموسم الأول 2008/2007 ـ أما في الموسم الثاني فكان إندان

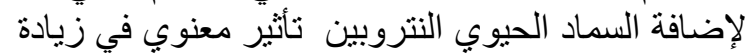

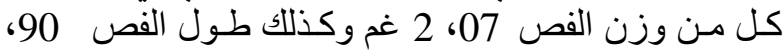
2 سم و TSS TS 91، 20 \% \% و النسبة المئوية للمادة الجافة 40، 46\% مقارنة بمعاملة الإضافة ، أما بالنسبة لتأثير

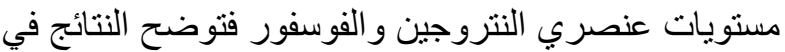
الجدول بان لمعاملات الإضافة تأثير معنوي في زئرئ زيادة قيم

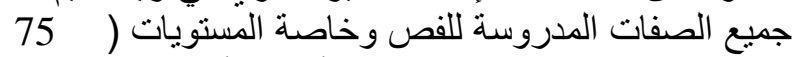
نتروجين و 150 فوسفور ) والتي أعطت أعلى القيم

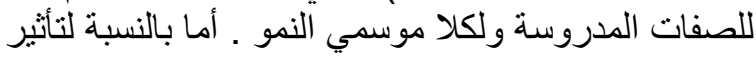

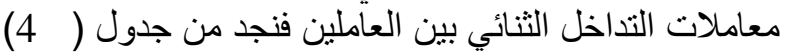

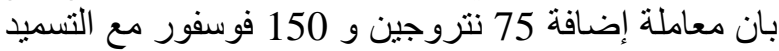

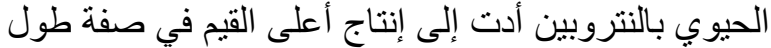
الفص وقطر الفص و النسبة المئوية للمادة الجافة وللمئ اللموسم الفي الأول أما أعلى وزن للفص 1 41، 2 غم فكان عند معاملة التداخل بين 50 نتروجين و 100 فوسفور مع اللمعين التسميد الحيوي .بينما أدت معاملة إضافة

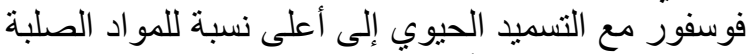

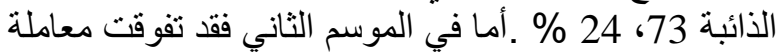

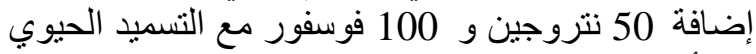

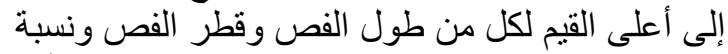

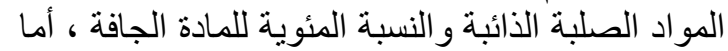

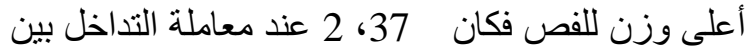
إضافة 75 نتروجين و 150 فوسفور وبدون

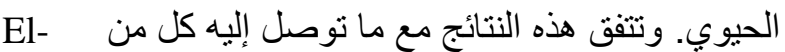

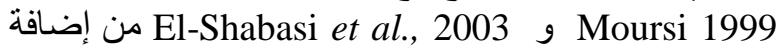
الأسمدة النتروجينية و الفوسفورية إلى نبات الثوم سواءً

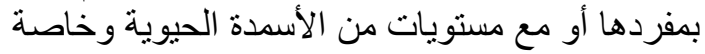

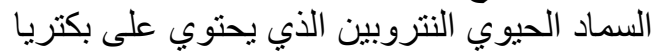

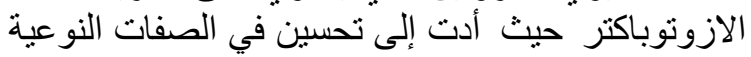

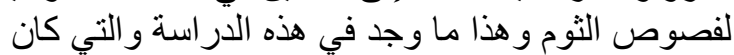

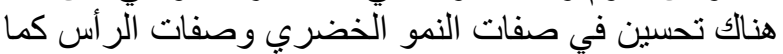

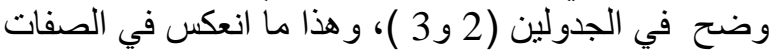

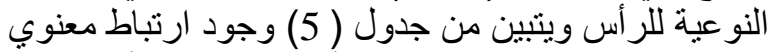

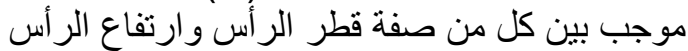

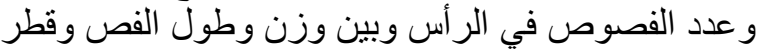

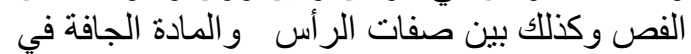
الفصوص وكذلك المواد الصلبة الذائبة في الفص لكلا

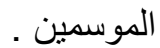
4-3-3 الإنتاج الكلي لوحدة المساحة

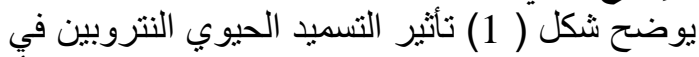

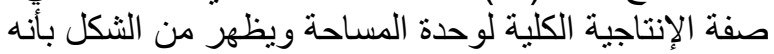

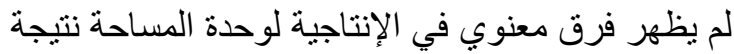

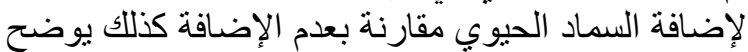

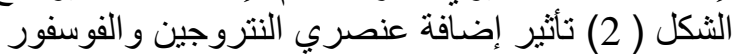
ويتضح بان أعلى إنتاجية كانت 13,12 و 24، 10 
and plant chemical composition .Zagazig J. Agric. Res. 31(4A): 1425-1440.

Bardisi A., El-Mansi A.A., Fayad A.N. and Abou El-Khair E.E. (2004 b). Effect of mineral NP and Boifertilizers on garlic under sandy soil conditions, B-Yield ,bulb quality and storability .Zagazig J. Agric. Res. 31(4A): 1441-1462.

Bashan Y. and Holguin G. (1997). Azospirillum plant relationship environmental and physiological advances (1990-1996). Can. J. Microbial 43:103-121.

Bhonde S. R., Sharma S.B. and Chougule A.B.(1997). Effect of biofertilizer in combination with nitrogen sources on yield and quality of onion .National Hort. Res. and Develop. Found. 17(2): 1-3.

El-Moursi A. H. A. (1999). Effect of some intercropping systems and nitrogen levels on growth yield and its components in garlic (Allium sativum L.) . Ph. D. Thesis, Fac. Agric., Mansoura Univ. 197 pp.

El-Seifi S. K. , Sawsan M.M. and Abdel- Fattah A.I. and Mohamed M.A.(2004). Effect of biofertilizers and nitrogen levels on the productivity and quality of chinese garlic under sandy soil conditions .Zagazig J. A gric. Res. 81(3): 889- 914.

El-Shabasi M. S. S., Gaafor S.A. and Zahran F. A. (2003). Efficiency of biofertilizer $\mathrm{Nr}$ under different levels of inorganic nitrogen fertilizer on growth, yield and its chemical constituents of garlic plants . J. Agric. Sci. , Mansoura Univ. 28(9):6927 6938.

Forlain G. M. , Branzani M., Pastorelli R. and Sarvilli S.(1995). Root potentially related properties in plant associated bacteria . J. of General Breeding Italy, 49(4): 343352.

Gardener F. D., Pearce R.B. and Mitchell R.L. (1985). Physiology of crop plants .The Iowa State Univ. Press. 327 pp.

Haller T. and Stople H.(1985). Quantitative estimation of root exudation of maize . Plant Soil, 86: 207- 216.

Kilgori M. J., Magaj M.D. and Yakubu A.I.(2007). Productivity of two garlic (Allium sativum L.) cultivars as affected by different levels of nitrogen and phosphorous fertilizers in Sokoto Nigeria. American -Eurasian J. Agric. and environ. Sci. ,2(2): 158- 162.

SAS (1996). Statistical Analysis System .SAS. Instiute. Inc. Cary, NC. 27511 U.S.A.

Silvia G. and Lipiski V.M.(2008).Effect of nitrogrn fertilization on yield and color of red garlic (Allium sativum L.) cultivars cien .Inv. Agri. 35(1): 57- 64. www. rcia puc. d.

Tien T. M., Gaskins M.H. and Hubble D.H.(1979). Plant growth substances produced by Azospirillum barasilense and their effect on growth of plants . Appl. Envirom. Microb. 37: 1016- 1024.

Tomas L. and Kielian B.W.(2006). Fertilization of garlic (Allium sativum L.) with nitrogrn and sulphur . Annales Universitatis Mariae Curie Sklodowska Lublin -Polonia. Annales Umes. Sec. E. 2006: 45- 50.

Xiaohongx T. and Masahiko M.S. (2002). Merits, utilization, perspectives of controlled release nitrogen fertilizers.Tohoku J. of Agric. Res. (23):67-72 
